\title{
Erratum to: Bandwidth enhancement of transformation optics-based cloak with reduced parameters
}

\author{
Archana Rajput $^{1} \cdot$ Kumar Vaibhav Srivastava ${ }^{1}$
}

Published online: 9 June 2015

(c) Springer-Verlag Berlin Heidelberg 2015

Erratum to: Appl. Phys. A

DOI 10.1007/s00339-015-9235-8

The original version of this article unfortunately contained a mistake.

The presentation of Eqs. (1)-(3) was incorrect. The correct versions are given below:

$\mu_{\mathrm{r}}=\left(\frac{r-a^{\prime}}{r}\right)^{2}, \quad \mu_{\theta}=1, \quad \epsilon_{z}=\left(\frac{b}{b-a^{\prime}}\right)^{2}$ $\mu_{\mathrm{r}}=\left(\frac{r-a^{\prime}}{r}\right)^{2}, \quad \mu_{\theta}=1, \quad \epsilon_{z}=\left(\frac{b}{b-\bar{a}}\right)^{2}$

$$
n_{\mathrm{r}}=\left(\frac{b}{b-\bar{a}}\right), \quad n_{\theta}=\left(\frac{r-a^{\prime}}{r}\right)\left(\frac{b}{b-\bar{a}}\right)
$$

The online version of the original article can be found under doi:10.1007/s00339-015-9235-8.

Archana Rajput

rajput.archana07@gmail.com

Kumar Vaibhav Srivastava

kvs@iitk.ac.in

1 Department of Electrical Engineering, Indian Institute of Technology Kanpur, Kanpur, India 